\title{
Bennett 机构交错角区间和机构类型的关系*
}

\author{
鹿 玲 ${ }^{1,2}$ 张一同 1,3 牟德君 ${ }^{1,3}$ 胡 波 ${ }^{1,3}$ 卢文娟 ${ }^{1,3}$ \\ (1. 燕山大学机械工程学院 秦皇岛 066004; \\ 2. 燕山大学河北省重型智能制造装备技术创新中心 秦皇岛 066004; \\ 3. 燕山大学河北省并联机器人与机电系统实验室 秦皇岛 066004)
}

\begin{abstract}
摘要: 目前 Bennett(BNT)机构根据回转副轴线的方向, 分成非对称安装和线性对称安装两类。为了研究这两类机构的共性和 区别, 在杆件交错角 $(0,2 \pi)$ 的区域内, 用相邻象限组合为 4 个区间, 由 $\mathrm{S}_{12}$ (第 1-2 象限)区间和 $\mathrm{S}_{34}$ (第 3-4 象限)区间各得到 1 组非对称安装的左旋和右旋 BNT 机构; 由 $\mathrm{S}_{23}$ (第 2-3 象限)和 $\mathrm{S}_{41}$ (第 4-1 象限)区间各得到 1 组线性对称安装的左旋和右旋 BNT 机构。把前后 2 组机构进行对比, 只有轴线方向是非对称的, 机构结构本身都是对称的。由于 $S_{12}$ 和 $S_{34}$ 区间的非对称安装机 构, 通过交错角的设定, 可以转换为 $\mathrm{S}_{41}$ 区间的线性对称机构, 所以线性对称和非对称之间并无本质差别, 4 组机构本质是 相同的 1 组。因为 $\mathrm{S}_{41}$ 区间机构的运动副的轴线方向指向相同, 轴线的正方向与相邻杆内角逆时针的角位移方向一致, 所以 把 $\mathrm{S}_{41}$ 区间改用交错角锐角表示的 $\mathrm{R}_{41}$ (第 4-1 象限)区间作为交错角的工作区间, 就得到线性对称的右旋型和左旋型两种 BNT 机构基本类型, 这两类机构的运动链是相同的, 只是以不同构件为机架得到两种机构的形式。根据这些概念, 左旋型和右 旋型 BNT 机构, 采用交错角锐角给出了统一的结构参数方程和含相邻杆内角表示的闭环位移方程。这种交错角为锐角的 统一参数方程, 简单, 直观, 便于应用。研究强调并补充了相邻杆的交错角不能在作用区间的同一象限选取的一个必要的 约束条件。
\end{abstract}

关键词: Bennett 机构; 约束条件; 交错角; 结构参数; 闭环位移方程

中图分类号: TH112

\section{Relationship between Stagger Angle Interval and Mechanism Type of the Bennett Mechanism}

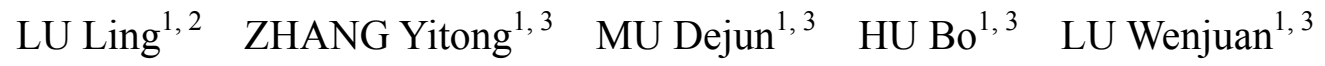 \\ (1. School of Mechanical Engineering, Yanshan University, Qinhuangdao 066004; \\ 2. Heavy-duty Intelligent Manufacturing Equipment Innovation Center of Hebei Province, \\ Yanshan University, Qinhuangdao 066004; \\ 3. Hebei Provincial Key Laboratory of Parallel Robot and Mechatronic System, \\ Yanshan University, Qinhuangdao 066004)
}

\begin{abstract}
At present, Bennett (BNT) mechanism can be divided into two types according to the direction of axis of revolute joints: Asymmetrical and linear symmetrical installation. In order to study the similarities and differences between these two types of mechanisms, using adjacent quadrants were used to form four intervals in the region of link stagger angle $(0,2 \pi)$. Then, a group of asymmetrically installed left-handed and right-handed BNT mechanisms are obtained based on the $\mathrm{S}_{12}$ (quadrant I and II) and $\mathrm{S}_{34}$ (quadrant III and IV)intervals, and a group of linearly symmetrically installed left-handed and right-handed BNT mechanisms are obtained based on the $\mathrm{S}_{23}$ (quadrant II and III)and $\mathrm{S}_{41}$ (quadrant IV and I)intervals. Comparing these two groups of mechanisms, only the axis is asymmetrical while the structure itself is symmetrical. Since the asymmetrical installation mechanisms in the regions of $\mathrm{S}_{12}$ and $\mathrm{S}_{34}$ can be transformed into linear symmetrical mechanisms in the region of $\mathrm{S}_{41}$ by setting the stagger angle, there is no essential difference between linear symmetrical and asymmetrical mechanisms, and these two groups of mechanisms are essentially the same one. Because the joints within the mechanism in the region of $\mathrm{S}_{41}$ have the same axial direction, and the positive direction of the axis is the same as the anti-clockwise angular displacement direction of the inner angle of the adjacent links, so the interval of
\end{abstract}

* 河北省自然科学基金(E2011203193)和河北省杰出青年基金 (E2017203094)资助项目。20190612 收到初稿, 20191124 收到修改稿 
$\mathrm{R}_{41}$ (quadrant IV and I)represented by the sharp angle of the stagger angle is used as the working interval of the stagger angle, then two basic types of BNT linear symmetrical mechanisms, i.e., right-handed and left-handed, are obtained. The kinematic chains of these two types of mechanisms are the same, but they are obtained by taking different components as the frame. According to these concepts, aiming to the left-handed and right-handed BNT mechanisms, the uniform structural parameter equations and the closed-loop displacement equations with the inner angles of adjacent links are given by using the stagger sharp angles. This unified parametric equation with sharp angles is simple, intuitive and easy to apply. A necessary constraint condition that the stagger angles of adjacent links cannot be selected in the same quadrant of the action region is emphasized and supplemented.

Key words: Bennett mechanism; constraint condition; stagger angle; structural parameters; closed-loop displacement equation

\section{0 前言}

BNT 机构是 1903 年英国科学家 BENNETT ${ }^{[1-2]}$ 提出的。它由 4 个 $2 \mathrm{R}$ 杆通过回转副首尾相接组成, 每个杆两端的回转副轴线的交错角不为零。由于机 构约束条件的特殊性, 使得机构的性能很复杂, 因 此机构学者们对其机构的自由度、各种特性和应用 进行了大量的研究 ${ }^{[3-13]}$ 。

BNT 机构杆件交错角的传统取值区 间 ${ }^{[14-16]}$ 为 $\left(0^{\circ}, 180^{\circ}\right)$, 由于轴线方向的不对称, 称之为非对称 安装。文献[17]提出了 BNT 机构的一种线性对称的 安装结构形式, 但是现有的文献都没有详细地讨论 相邻杆件交错角的所有作用区间与 BNT 机构的非 线性安装和线性对称的安装之间的内在关系。

为了能更清晰地了解它们之间的内在关系, 以 及它们与 BNT 机构类型的关系, 本文下面将对这 个问题进行详细讨论。并且给出 BNT 机构两种结 构形式、结构参数和闭环位移参数方程的统一的表 达形式。

\section{1 交错角区间与机构类型的关系}

图 1 和图 2 是 $\mathrm{BNT}$ 机构的一个 $2 \mathrm{R}$ 杆件的两个 视图, 两轴 $A$ 和 $B$ 的交错角为 $\alpha$, 逆时针为正, 顺 时针为负。为了叙述方便, 规定在 BNT 机构中, 相 邻杆的一个杆长 $a$ 的交错角为 $\alpha$, 另一个杆长 $b$ 的 交错角为 $\beta$ 。

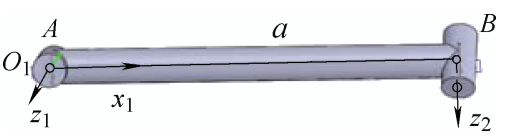

图 $12 \mathrm{R}$ 杆正面

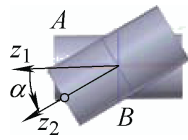

图 2 侧视图
BNT 机构杆件交错角的传统取值区间为 $\left(0^{\circ}\right.$, $\left.180^{\circ}\right)$, 称之为交错角的第 $\mathrm{S}_{12}$ 作用区间, 简称交错 角的 $\mathrm{S}_{12}$ 区间。本文提出相邻杆交错角的一个必要 几何约束条件：相邻杆的交错角不能在作用区间的 同一象限选取, 简称相邻杆交错角的异象限约束条
件。在 $\mathrm{S}_{12}$ 区间, 当机架的交错角为第一象限 $0^{\circ}<\beta<90^{\circ}$ 时，连架杆的交错角必须在第二象限选取 $90^{\circ}<\alpha<180^{\circ}$ 。这样在 $\mathrm{S}_{12}$ 区间得到图 3 非对称安装的 右旋型(连杆相对机架成右螺旋型)BNT 机构, 连杆 $B C$ 相对机架 $A D$ 成右旋状态。

当 $90^{\circ}<\beta<180^{\circ} ， 0^{\circ}<\alpha<90^{\circ}$ 时，在 $\mathrm{S}_{12}$ 区间得到 图 4 非对称安装的左旋型 $\mathrm{BNT}$ 机构, 连杆 $B C$ 相对 机架 $A D$ 成左旋状态。
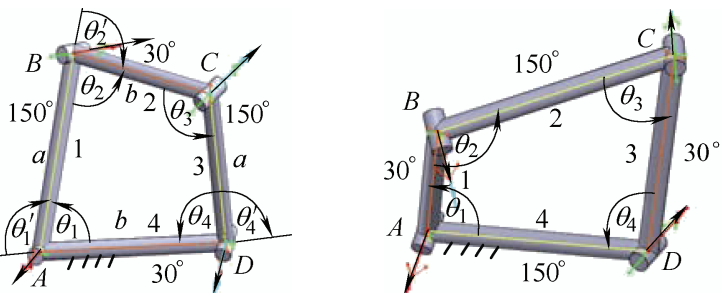

图 $3 \quad \mathrm{~S}_{12}$ 区右旋型非对称安装 图 $4 \mathrm{~S}_{12}$ 区左旋型非对称安装

这两种类型由于运动副的轴线 $z$ 方向不对称, 称为非对称安装(非对称是指运动副的轴线方向不 对称)。文献[1-2,14-16]给出的 BNT 机构都是非对称 的传统安装方式。

图 5a 是文献[17]给出的一种非对称安装的 BNT 机构, 显然图 5a 就是图 3 或图 4 的一种。文献[17] 在文献[18]的基础上, 通过对一个构件的 $180^{\circ}$ 翻转, 改变了该构件两个回转副的轴线方向，使得变化后 机构运动副的轴线实现了对称, 得到了图 $5 \mathrm{~b}$ 的线性 对称的安装结构形式 ${ }^{[18-20]}$ 。

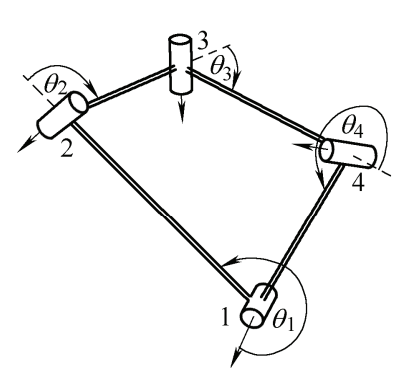

(a) 非对称安装

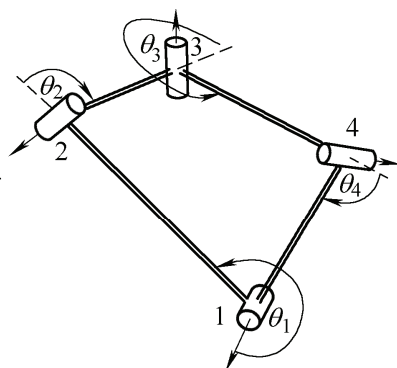

(b) 线性对称安装
图 5 现有文献及文献[17]对 BNT 机构的分类

相邻杆交错角在作用域内的一个周期为 $\left(0^{\circ}\right.$, $\left.360^{\circ}\right)$, 可以任意选两个相邻的象限作为它的一个作 用区间，这样可以得到 4 个作用区间：第一个作用 
区间 $\mathrm{S}_{12}$ 为第一象限和第二象限 $\left(0^{\circ}, 180^{\circ}\right)$; 第二个 作用区间 $S_{23}$ 区为第二象限和第三象限 $\left(90^{\circ}, 270^{\circ}\right)$; 第三个作用区间 $\mathrm{S}_{34}$ 区为第三象限和第四象限 $\left(180^{\circ}\right.$, $\left.360^{\circ}\right)$; 第四个作用区间 $\mathrm{S}_{41}$ 为第四象限和第一象限 $\left(270^{\circ}, 90^{\circ}\right)$ 。

选取杆件的交错角时, 必须充分注意到象限约 束条件: 相邻杆的交错角不能在区间内的同一象限 选取。任选一个作用区间, 都可以得到以机架为参 考系的两种类型的 BNT 机构: 左旋型 BNT 机构和 右旋型 BNT 机构。

\section{1 相邻杆交错角的 $S_{12}$ 区间}

根据相邻杆交错角的异象限约束条件, 在相邻 杆交错角的 $\mathrm{S}_{12}$ 区间 $\left(0^{\circ}, 180^{\circ}\right)$ 内, 交错角的选取方案 有两种: (1) $0^{\circ}<\beta<90^{\circ}, 90^{\circ}<\alpha<180^{\circ}$; (2) $0^{\circ}<\alpha<90^{\circ}$, $90^{\circ}<\beta<180^{\circ}$ 。对应这两种交错角的选择, 分别得到 图 3 的 $\mathrm{S}_{12}$ 区右旋型和图 4 的 $\mathrm{S}_{12}$ 区左旋型的两种非 对称安装的机构。图 3 的交错角为 $\beta=30^{\circ}, \alpha=150^{\circ}$, 图 4 为 $\beta=150^{\circ}, \alpha=30^{\circ}$; 两图的杆长 $a=b=120 \mathrm{~mm}$ 。

在图 3 的 $\mathrm{S}_{12}$ 区右旋型 BNT 机构, 机架的两个 轴线都指向前方, 方向相同, 连杆的两个轴线都指 向后方, 方向相同。在图 4 的 $\mathrm{S}_{12}$ 区左旋型 $\mathrm{BNT}$ 机 构, 连架杆 $A B$ 的两个轴线都指向前方, 方向相同。 连架杆 $C D$ 的两个轴线都指向后方, 方向相同。

\section{2 相邻杆交错角的 $S_{23}$ 区间}

在 $\mathrm{S}_{23}$ 区间 $\left(90^{\circ}, 270^{\circ}\right)$ 内, 相邻杆交错角的选取 方案有两种: (1) $180^{\circ}<\beta<270^{\circ}, 90^{\circ}<\alpha<180^{\circ}$; (2) $90^{\circ}<\beta<180^{\circ}, 180^{\circ}<\alpha<270^{\circ}$ 。对应这两种交错角的选 择, 分别得到图 6 的 $\mathrm{S}_{23}$ 区右旋型 BNT 机构和图 7 的 $\mathrm{S}_{23}$ 区左旋型 BNT 机构, 两种都是线对称安装的 BNT 机构。

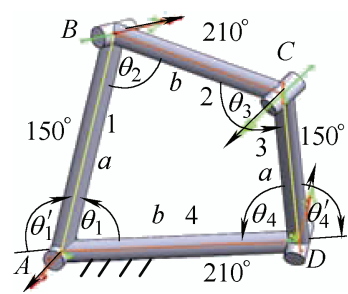

图 $6 \mathrm{~S}_{23}$ 区右旋型

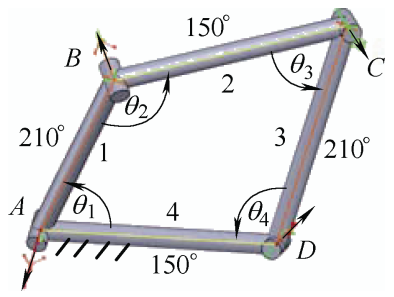

图 $7 \mathrm{~S}_{23}$ 区左旋型
图 6 的交错角为 $\beta=210^{\circ}, \alpha=150^{\circ}$, 图 7 为 $\beta=150^{\circ}, \alpha=210^{\circ}$; 两图的杆长 $a=b=120 \mathrm{~mm}$ 。

在图 6 的 $\mathrm{S}_{23}$ 区右旋型 $\mathrm{S}_{23}$ 中, 所有杆的两个轴 线都指向相反。在图 7 的 $S_{23}$ 区左旋型中, 所有杆 的两个轴线都指向相反。

\section{3 相邻杆交错角的 $S_{34}$ 区间}

在 $\mathrm{S}_{34}$ 区间 $\left(180^{\circ}, 360^{\circ}\right)$ 内, 相邻杆交错角的选 取方案有两种: (1) $180^{\circ}<\beta<270^{\circ}, 270^{\circ}<\alpha<360^{\circ}$; (2) $270^{\circ}<\beta<360^{\circ}, 180^{\circ}<\alpha<270^{\circ}$ 。对应这两种交错角的 选择, 分别得到图 8 的 $\mathrm{S}_{34}$ 区右旋型 BNT 机构和图 9 的 $\mathrm{S}_{34}$ 区左旋型 BNT 机构, 两种都是线对称安装 的 BNT 机构。图 8 的交错角为 $\beta=210^{\circ}, \alpha=330^{\circ}$, 图 9 为 $\beta=330^{\circ}, \alpha=210^{\circ}$; 两图的杆长 $a=b=120 \mathrm{~mm}$ 。

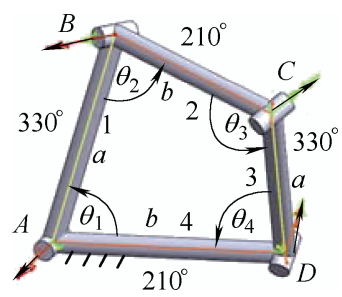

图 $8 \mathrm{~S}_{34}$ 区右旋型

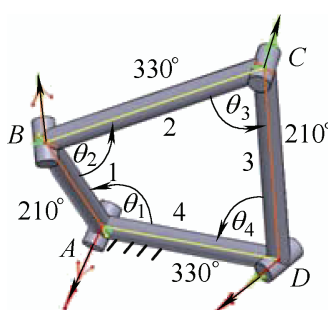

图 $9 \mathrm{~S}_{34}$ 区左旋型
在图 8 的 $\mathrm{S}_{34}$ 区右旋型 BNT 机构中, 机架的两 个轴线指向相反, 连杆的两个轴线指向相反, 连架 杆 $A B$ 的两个轴线都指向前方, 方向相同。连架杆 $C D$ 的两个轴线都指向后方，方向相同。

在图 9 的 $\mathrm{S}_{34}$ 区左旋型 BNT 机构中, 连架杆 $A B$ 的两个轴线指向相反。连架杆 $C D$ 的两个轴线指 向相反。机架的两个轴线都指向前方, 方向相同。 连杆的两个轴线都指向后方, 方向相同。

\section{4 相邻杆交错角的 $\mathrm{S}_{41}\left(\mathrm{R}_{41}\right)$ 区间}

在 $\mathrm{S}_{41}$ 区间的第 4 和 1 象限 $\left(270^{\circ}, 90^{\circ}\right)$ 内, 相邻 杆交错角的选取方案有两种： (1) $0^{\circ}<\beta<90^{\circ}$, $270^{\circ}<\alpha<360^{\circ}$; (2) $270^{\circ}<\beta<360^{\circ}, 0^{\circ}<\alpha<90^{\circ}$ 。对应这 两种交错角的选择, 分别得到图 10 的 $\mathrm{S}_{41}$ 区右旋型 BNT 机构和图 11 的 $\mathrm{S}_{41}$ 区左旋型 BNT 机构。

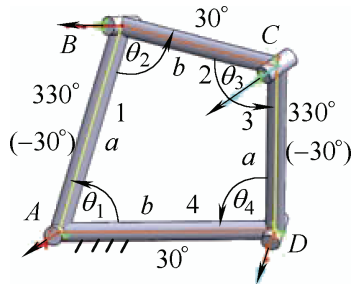

图 $10 \mathrm{~S}_{41}\left(\mathrm{R}_{41}\right)$ 区右旋型

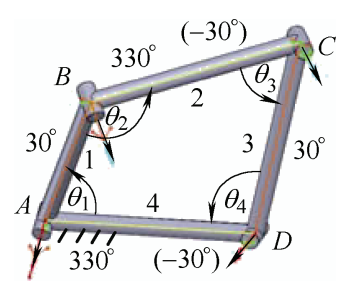

图 $11 \mathrm{~S}_{41}\left(\mathrm{R}_{41}\right)$ 区左旋型
图 10 的交错角为 $\beta=30^{\circ}, \alpha=330^{\circ}$, 图 11 为 $\beta=330^{\circ}, \alpha=30^{\circ}$; 两图的杆长 $a=b=120 \mathrm{~mm}$ 。

这两种机构都是线对称安装的, 所有杆的两个 轴线都指向前方，方向相同。

相邻杆交错角的第 $\mathrm{S}_{41}$ 区间 $\left(270^{\circ}, 90^{\circ}\right)$ ，用交错 角的锐角表示为 $\left(-90^{\circ}, 90^{\circ}\right)$, 简称为 $\mathrm{R}_{41}$ 区间。在 $\mathrm{R}_{41}$ 区 $\left(-90^{\circ}, 90^{\circ}\right)$ 中, $\mathrm{R}_{41}$ 区右旋型 $\mathrm{BNT}$ 机构的交错 角取值为 $-90^{\circ}<\alpha<0^{\circ}, 0^{\circ}<\beta<90^{\circ} ; \mathrm{R}_{41}$ 区左旋型 BNT 机构交错角的取值为 $0^{\circ}<\alpha<90^{\circ},-90^{\circ}<\beta<0^{\circ}$ 。

例如图 10 中 $\mathrm{R}_{41}$ 区的右旋型 $\mathrm{BNT}$ 机构交错角 为 $\beta=30^{\circ}, \alpha=30^{\circ}$, 图 $11 \mathrm{R}_{41}$ 区的左旋型 $\mathrm{BNT}$ 机构 为 $\beta=30^{\circ}, \alpha=30^{\circ}$ 。 


\section{2 非对称安装和线性对称安装}

图 5a 就是文献[17]给出的一种非对称安装的 BNT 机构, 所谓非对称安装是指运动副的轴线指向 (即所规定的轴线正向)是非对称的。例如图 $5 \mathrm{a}$ 中, $z_{1}$ 轴和 $z_{3}$ 轴方向不是线性对称的, $z_{2}$ 轴和 $z_{4}$ 轴方向 也不是线性对称的。

交错角为 $\mathrm{S}_{12}$ 区的传统 BNT 机构和交错角为 $\mathrm{S}_{34}$ 区的 BNT 机构都是非线性安装的。为了确保 BNT 机构得到封闭环, 非对称安装必须遵守 “邻杆交错 角的异象限约束条件”, 否则机构不能封闭。这是机 构封闭的必要条件之一。

根据交错角的异象限约束条件, 在 $\mathrm{S}_{12}$ 区的 $\left(0^{\circ}\right.$, $\left.180^{\circ}\right)$ 内, 相邻杆交错角可以有两种选取方案: (1) $0^{\circ}<\beta<90^{\circ}, 90^{\circ}<\alpha<180^{\circ}$; (2) $0^{\circ}<\alpha<90^{\circ}, 90^{\circ}<\beta<180^{\circ}$ 。 对应这两种交错角的选择分别得到图 3 左旋型和图 4 右旋型的两种非对称安装的机构。在 $\mathrm{S}_{34}$ 区的 $\left(180^{\circ}, 360^{\circ}\right)$ 内, 相邻杆交错角可以有两种选取方案: (1) $180^{\circ}<\beta<270^{\circ}, 270^{\circ}<\alpha<360^{\circ}$; (2) $270^{\circ}<\beta<360^{\circ}$, $180^{\circ}<\alpha<270^{\circ}$ 。

\section{1 非对称安装的结构参数和闭环位移方程}

\subsection{1 非对称安装结构参数}

由前面的分析知, 相邻杆交错角为 $S_{12}$ 和 $S_{34}$ 区 间时, 得到非对称安装 BNT 机构。非对称安装 BNT 的结构参数几何约束条件是: (1) 对边的杆长相等和 交错角相等, 交错角的旋向相同; (2) 相邻两个边长 比值和对应交错角正弦的比值相等, 即

$$
\begin{gathered}
a_{1}=a_{3}=a \quad b_{2}=b_{4}=b \quad \alpha_{1}=\alpha_{3}=\alpha \quad \beta_{2}=\beta_{4}=\beta \\
\frac{a}{b}=\frac{\sin \alpha}{\sin \beta}
\end{gathered}
$$

式(2)是相邻杆的比例方程。当限定交错角 $0<\alpha<360^{\circ}$ 时, 式(2)仅仅适合 $\mathrm{S}_{12}$ 区和 $\mathrm{S}_{34}$ 区的 BNT 机构。

2.1.2 非对称安装的闭环位移方程

由文献 17 的图 5a 可以得出, 非对称 BNT 机构 的位移方程为

$$
\begin{gathered}
\theta_{1}^{\prime}+\theta_{3}^{\prime}=2 \pi \quad \theta_{2}^{\prime}+\theta_{4}^{\prime}=2 \pi \\
\tan \frac{\theta_{1}^{\prime}}{2} \tan \frac{\theta_{2}^{\prime}}{2}=\frac{\sin \frac{\beta+\alpha}{2}}{\sin \frac{\beta-\alpha}{2}}=k
\end{gathered}
$$

式(4)称之为相邻杆外角的正切积的正弦形式, $k$ 为 外角的正切积的正弦形式常数, 它只适合 $S_{12}$ 区和 $\mathrm{S}_{34}$ 区的 BNT 机构。式(3)和式(4)中 $\theta_{1}^{\prime} 、 \theta_{2}^{\prime} 、 \theta_{3}^{\prime}$ 和 $\theta_{4}^{\prime}$ 都是相邻杆的外角, $\theta_{1} 、 \theta_{2} 、 \theta_{3}$ 和 $\theta_{4}$ 是相邻杆的内 角, 如图 3 所示。并且 $\theta_{1}^{\prime}=\pi-\theta_{1}, \theta_{2}^{\prime}=\pi-\theta_{2}$, $\theta_{3}^{\prime}=\pi-\theta_{3}, \quad \theta_{4}^{\prime}=\pi-\theta_{4}, \quad \theta_{2}^{\prime}=\theta_{4}^{\prime}$ 。把 $\theta_{1}^{\prime}=\pi-\theta_{1}$, $\theta_{2}^{\prime}=\pi-\theta_{2}$, 代入式(4), 得

$$
\tan \frac{\pi-\theta_{1}}{2} \tan \frac{\pi-\theta_{2}}{2}=\arctan \frac{\theta_{1}}{2} \arctan \frac{\theta_{2}}{2}=\frac{\sin \frac{\beta+\alpha}{2}}{\sin \frac{\beta-\alpha}{2}}
$$

$$
\tan \frac{\theta_{1}}{2} \tan \frac{\theta_{2}}{2}=\frac{\sin \frac{\beta-\alpha}{2}}{\sin \frac{\beta+\alpha}{2}}=k
$$

式(5)称之为相邻杆内角的正弦形正切积方程, $k$ 为 内角的正弦形正切积常数。式(5)适用于 $S_{12}$ 和 $S_{34}$ 区间。

\section{2 线性对称安装的结构参数和闭环位移方程}

文献[17]在文献[18]的基础上，通过一个构件 $180^{\circ}$ 的翻转, 改变了回转副的轴线方向, 使机构的 运动副轴线实现了线性对称安装。

由前面的分析知, $\mathrm{S}_{23}$ 区左旋型和右旋型 BNT 机构, 以及 $\mathrm{S}_{41}$ 区左旋型和右旋型 BNT 机构, 都是 线对称的 BNT 机构。

\subsection{1 线性对称安装的结构参数}

线性对称安装的 BNT 机构的结构参数几何约 束条件是: (1) 对边的杆长相等和交错角相等, 交错 角的旋向相同; (2) 相邻两边的边长比值与交错角的 正弦比值相等, 符号相反。即

$$
\begin{gathered}
a_{1}=a_{3}=a \quad b_{2}=b_{4}=b \quad \alpha_{1}=\alpha_{3}=\alpha \quad \beta_{2}=\beta_{4}=\beta \\
\frac{a}{b}=-\frac{\sin \alpha}{\sin \beta}
\end{gathered}
$$

限定交错角 $0<\alpha<360^{\circ}$ 时, 式(7)仅仅适合 $\mathrm{S}_{23}$ 区和 $\mathrm{S}_{41}$ 区的 BNT 机构。因为在 $\mathrm{S}_{23}$ 区和 $\mathrm{S}_{41}$ 区，交错角 的约束法则使 $\sin \alpha$ 或者 $\sin \beta$ 必有一个是小于零 的, 所以 $(-\sin \alpha / \sin \beta)>0$ 。

根据 BNT 机构相邻杆交错角的异象限约束法 则, 在 $S_{23}$ 区, 当 $180^{\circ}<\beta<270^{\circ}, 90^{\circ}<\alpha<180^{\circ}$ 时, 得 到图 6 的右旋型 $\mathrm{BNT}$ 机构, 当 $90^{\circ}<\beta<180^{\circ}$, $180^{\circ}<\alpha<270^{\circ}$ 时, 得到图 7 的左旋型 $\mathrm{BNT}$ 机构。

在 $\mathrm{S}_{41}$ 区, 当 $0^{\circ}<\beta<90^{\circ}, 270^{\circ}<\alpha<360^{\circ}$ 时, 得到 图 10 的右旋型 $\mathrm{BNT}$ 机构; 当 $270^{\circ}<\beta<360^{\circ}$, $0^{\circ}<\alpha<90^{\circ}$ (或者用锐角表示 $-90^{\circ}<\beta<0^{\circ}, 0^{\circ}<\alpha<90^{\circ}$ )时， 得到图 11 的左旋型 BNT 机构。

\subsection{2 线性对称安装的闭环位移方程}

线对称安装的 BNT 机构的位移方程为

$$
\begin{gathered}
\theta_{1}^{\prime}=\theta_{3}^{\prime} \quad \theta_{2}^{\prime}=\theta_{4}^{\prime} \\
\tan \frac{\theta_{1}^{\prime}}{2} \tan \frac{\theta_{2}^{\prime}}{2}=\frac{\cos \frac{\beta+\alpha}{2}}{\cos \frac{\beta-\alpha}{2}}=k
\end{gathered}
$$


式(9)称之为相邻杆的外角的正切积的余弦形式, $k$ 为外角的余正弦形切积常数, 它只适合 $\mathrm{S}_{23}$ 区和 $\mathrm{S}_{41}$ 区的 BNT 机构。式(8)和式(9)中 $\theta_{1}^{\prime} 、 \theta_{2}^{\prime} 、 \theta_{3}^{\prime}$ 和 $\theta_{4}^{\prime}$ 是 相邻杆的外角, $\theta_{1} 、 \theta_{2} 、 \theta_{3}$ 和 $\theta_{4}$ 是相邻杆的内角, 如图 6 所示。把 $\theta_{1}^{\prime}=\pi-\theta_{1}, \theta_{2}^{\prime}=\pi-\theta_{2}$, 代入式(9), 得

$$
\begin{gathered}
\tan \frac{\pi-\theta_{1}}{2} \tan \frac{\pi-\theta_{2}}{2}=\arctan \frac{\theta_{1}}{2} \arctan \frac{\theta_{2}}{2}=\frac{\cos \frac{\beta+\alpha}{2}}{\cos \frac{\beta-\alpha}{2}} \\
\tan \frac{\theta_{1}}{2} \tan \frac{\theta_{2}}{2}=\frac{\cos \frac{\beta-\alpha}{2}}{\cos \frac{\beta+\alpha}{2}}=k
\end{gathered}
$$

式(10)称之为相邻杆内角的正切积的余弦形式, $k$ 为 内角的正切积的余弦形式常数。式(10)适用于 $S_{23}$ 、 $\mathrm{S}_{41} 、 \mathrm{R}_{41}$ 区间。

\section{3 非对称和线对称安装的本质属性}

\section{1 非对称和线对称安装具有结构相同的本质属性}

在交错角 $(0,360)$ 区间内, 由 $S_{12}$ 和 $S_{34}$ 区间各得 到 1 组非对称安装的左旋型和右旋型 BNT 机构; 由 $S_{23}$ 和 $S_{41}$ 区间各得到 1 组线对称安装的左旋型和右 旋型 BNT 机构。

对比前 2 组机构和后 2 组机构, 可以看出非对 称的 BNT 机构与线性对称的 BNT 机构, 除了运动 副回转轴线的对称性有区别外, 机构结构本身都是 完全相同的, 都是线性对称的, 所以线性对称和非 对称之间并无本质差别, 因此从机械结构方面来讲 4 组机构本质是相同的 1 组机构。

从结构参数和位移参数方面来讲, 非对称和线 对称只有相邻杆的比例方程和正切积方程表达形式 的不同, 其余都相同。相邻杆的比例方程式(2)和式 (7), 只有一个正负号之差, 它是交错角在不同象限 的正负不同引起的, 它们的绝对值都是相同的, 所 以它们之间没用本质区别。

式(4)是外角正切积交错角的正弦形式, 式(9)是 外角正切积交错角的余弦形式, 虽然表达式略有区 别, 但是通过交错角象限的转换, 可以把外角正切积 的正弦形式转换为正切积的余弦形式。因此, 非对称 和线对称的正切积方程也没有本质区别的不同。

下面详细给出非对称正切积的交错角正弦形式 转换成正切积交错角为锐角余弦形式的过程, 以及 线对称正切积交错角为锐角的余弦形式表示。

\section{2 非对称和线对称安装正切积交错角的锐角表示}

下面给出非对称安装 $\mathrm{S}_{12}$ 区、 $\mathrm{S}_{34}$ 区和线对称安
装 $S_{23}$ 正切积正弦型转换为 $R_{41}$ 区的正切积余弦型的 过程。

3.2.1 $\mathrm{S}_{12}$ 区正切积正弦型转换为 $\mathrm{R}_{41}$ 区的余弦型

对于 $\mathrm{S}_{12}$ 区右旋型 $\mathrm{BNT}$ 机构, 由于 $0^{\circ}<\beta<90^{\circ}$, $90^{\circ}<\alpha<180^{\circ}$, 只要第二象限的 $90^{\circ}<\alpha<180^{\circ}$ 转化为第 四象限的锐角。如图 12 所示, 设 $-90^{\circ}<\alpha^{\prime}<0^{\circ}$, 用 $\alpha=\pi+\alpha^{\prime}$ 代入式(4)

$$
\begin{gathered}
\tan \frac{\theta_{1}^{\prime}}{2} \tan \frac{\theta_{2}^{\prime}}{2}=\frac{\sin \left(\frac{\beta+\pi+\alpha^{\prime}}{2}\right)}{\sin \left(\frac{\beta-\left(\pi+\alpha^{\prime}\right)}{2}\right)}=\frac{\sin \left(\frac{\pi}{2}+\frac{\beta+\alpha^{\prime}}{2}\right)}{\sin \left(-\frac{\pi}{2}+\frac{\beta-\alpha^{\prime}}{2}\right)} \\
\tan \frac{\theta_{1}^{\prime}}{2} \tan \frac{-\theta_{2}^{\prime}}{2}=\frac{\cos \left(\frac{\beta+\alpha^{\prime}}{2}\right)}{\cos \left(\frac{\beta-\alpha^{\prime}}{2}\right)}
\end{gathered}
$$

式(11)就是右旋型 BNT 机构, $\mathrm{S}_{12}$ 区外角正切积正弦 形转换到 $\mathrm{R}_{41}$ 区的外角正切积交错角为锐角的余弦 表达形式, 简称为 $R_{41}$ 区外角正切积余弦式。

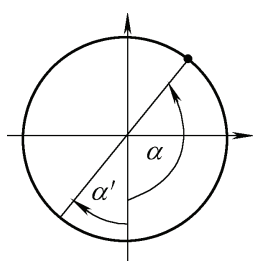

图 $122 \rightarrow 4$ 象限转换

对于 $\mathrm{S}_{12}$ 区左旋型 $\mathrm{BNT}$ 机构, $90^{\circ}<\beta<180^{\circ}$, $0^{\circ}<\alpha<90^{\circ}$ 。用 $\beta=\pi+\beta^{\prime}\left(-90^{\circ}<\beta^{\prime}<0^{\circ}\right)$ 代入式(4)

$$
\tan \frac{\theta_{1}^{\prime}}{2} \tan \frac{\theta_{2}^{\prime}}{2}=\frac{\sin \frac{\pi+\beta^{\prime}+\alpha}{2}}{\sin \frac{\pi+\beta^{\prime}-\alpha}{2}}=\frac{\cos \frac{\beta^{\prime}+\alpha}{2}}{\cos \frac{\beta^{\prime}-\alpha}{2}}
$$

即

$$
\tan \frac{\theta_{1}^{\prime}}{2} \tan \frac{\theta_{2}^{\prime}}{2}=\frac{\cos \frac{\beta^{\prime}+\alpha}{2}}{\cos \frac{\beta^{\prime}-\alpha}{2}}
$$

显然, 式(12)是 $\mathrm{R}_{41}$ 区用锐角 $\beta^{\prime}$ 和 $\alpha$ 表示的左旋型 BNT机构外角正切积的余弦形式。

\subsection{2 $\mathrm{S}_{34}$ 区正切积正弦型转换为 $\mathrm{R}_{41}$ 区的余弦型}

对于 $\mathrm{S}_{34}$ 区的右旋型BNT机构, $180^{\circ}<\beta<270^{\circ}$, $270^{\circ}<\alpha<360^{\circ}$ 。把 $\beta$ 和 $\alpha$ 分别由第二象和第三象限转换 为锐角表示的 $\mathrm{R}_{41}$ 区。如图 13 所示, 设 $\beta=\pi+\beta^{\prime}$ $\left(0^{\circ}<\beta^{\prime}<90^{\circ}\right), \alpha=2 \pi+\alpha^{\prime}\left(-90^{\circ}<\alpha^{\prime}<0^{\circ}\right)$, 代入式(4), 则

$$
\tan \frac{\theta_{1}^{\prime}}{2} \tan \frac{\theta_{2}^{\prime}}{2}=\frac{\sin \frac{\pi+\beta^{\prime}+2 \pi+\alpha^{\prime}}{2}}{\sin \frac{\pi+\beta^{\prime}-\left(2 \pi+\alpha^{\prime}\right)}{2}}=\frac{\cos \frac{\beta^{\prime}+\alpha^{\prime}}{2}}{\cos \frac{\beta^{\prime}-\alpha^{\prime}}{2}}
$$




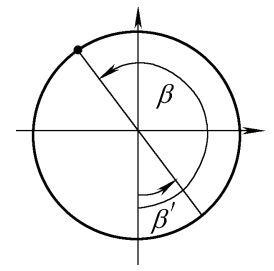

图 $13 \quad 3 \rightarrow 1$ 象限转换

对于 $\mathrm{S}_{34}$ 区左旋型 $\mathrm{BNT}$ 机构, $270^{\circ}<\beta<360^{\circ}$, $180^{\circ}<\alpha<270^{\circ}$ 。设 $\beta=2 \pi+\beta^{\prime}\left(-90^{\circ}<\beta^{\prime}<0^{\circ}\right), \alpha=\pi+\alpha^{\prime}$ $\left(0^{\circ}<\alpha^{\prime}<90^{\circ}\right)$, 代入式(4), 则

$$
\begin{gathered}
\tan \frac{\theta_{1}^{\prime}}{2} \tan \frac{\theta_{2}^{\prime}}{2}=\frac{\sin \frac{2 \pi+\beta^{\prime}+\pi+\alpha^{\prime}}{2}}{\sin \frac{2 \pi+\beta^{\prime}-\left(\pi+\alpha^{\prime}\right)}{2}}=-\frac{\cos \frac{\beta^{\prime}+\alpha^{\prime}}{2}}{\cos \frac{\beta^{\prime}-\alpha^{\prime}}{2}} \\
\tan \frac{\theta_{1}^{\prime}}{2} \tan \frac{-\theta_{2}^{\prime}}{2}=\frac{\cos \frac{\beta^{\prime}+\alpha^{\prime}}{2}}{\cos \frac{\beta^{\prime}-\alpha^{\prime}}{2}}
\end{gathered}
$$

\subsection{3 $\mathrm{S}_{23}$ 区正切积正弦型转换为 $\mathrm{R}_{41}$ 区的余弦型}

对于 $\mathrm{S}_{23}$ 区右旋型BNT机构, $180^{\circ}<\beta<270^{\circ}$, $90^{\circ}<\alpha<180^{\circ}$ 。设 $\beta=2 \pi+\beta^{\prime}\left(0^{\circ}<\beta^{\prime}<90^{\circ}\right)$, $\alpha=\pi+\alpha^{\prime}\left(-90^{\circ}<\alpha^{\prime}<0^{\circ}\right)$, 代入式(4), 则

$$
\begin{gathered}
\tan \frac{\theta_{1}^{\prime}}{2} \tan \frac{\theta_{2}^{\prime}}{2}=\frac{\sin \frac{2 \pi+\beta^{\prime}+\pi+\alpha^{\prime}}{2}}{\sin \frac{2 \pi+\beta^{\prime}-\left(\pi+\alpha^{\prime}\right)}{2}}=\frac{-\cos \frac{\beta^{\prime}+\alpha^{\prime}}{2}}{\cos \frac{\beta^{\prime}-\alpha^{\prime}}{2}} \\
\tan \frac{\theta_{1}^{\prime}}{2} \tan \frac{-\theta_{2}^{\prime}}{2}=\frac{\cos \frac{\beta^{\prime}+\alpha^{\prime}}{2}}{\cos \frac{\beta^{\prime}-\alpha^{\prime}}{2}}
\end{gathered}
$$

对于 $\mathrm{S}_{23}$ 区左旋型 $\mathrm{BNT}$ 机构, $180^{\circ}<\alpha<270^{\circ}$, $90^{\circ}<\beta<180^{\circ}$ 。设 $\alpha=2 \pi+\alpha^{\prime}\left(0^{\circ}<\alpha^{\prime}<90^{\circ}\right)$, $\beta=\pi+\beta^{\prime}\left(-90^{\circ}<\beta^{\prime}<0^{\circ}\right)$, 代入式(4), 则 $\tan \frac{\theta_{1}^{\prime}}{2} \tan \frac{\theta_{2}^{\prime}}{2}=\frac{\sin \frac{\pi+\beta^{\prime}+2 \pi+\alpha^{\prime}}{2}}{\sin \frac{\pi+\beta^{\prime}-\left(2 \pi+\alpha^{\prime}\right)}{2}}=\frac{\cos \frac{\beta^{\prime}+\alpha^{\prime}}{2}}{\cos \frac{\beta^{\prime}-\alpha^{\prime}}{2}}$

\subsection{4 $\mathrm{S}_{41}$ 和 $\mathrm{R}_{41}$ 区外角正切积的余弦形式}

由于 $S_{41}$ 区和 $R_{41}$ 区是同一个区域。所以计算该 区域的外角正切积, 直接采用 $\mathrm{R}_{41}$ 区的外角正切积 余弦形式的式(16)即可。

由上面的分析可知, 非线性的 $\mathrm{S}_{12} 、 \mathrm{~S}_{34}$ 区和线 对称的 $\mathrm{S}_{23}$ 区都可以通过交错角象限的变换, 转换 到用锐角表示的 $\mathrm{R}_{41}$ 区, 在 $\mathrm{R}_{41}$ 区使外角正切积用交 错角的余弦表达形式。因此, 从本质上讲, 非对称 安装和线对称安装是同一种机构, 只不过正切积的 表达形式不同。因此，在 $\mathrm{R}_{41}$ 区用交错角为锐角的 $\alpha$ 取代 $\alpha^{\prime}, \beta$ 取代 $\beta^{\prime}$ ，相邻杆外角为 $\theta_{1}{ }^{\prime} 、 \theta_{2}{ }^{\prime}$ (用 $\theta_{2}{ }^{\prime}$ 取代 $\theta_{2}{ }^{\prime}$ 和 $\left.-\theta_{2}{ }^{\prime}\right)$, 就可以得到相邻杆外角统一的正切积交
错角为锐角的余弦表达式, 即

$$
\tan \frac{\theta_{1}^{\prime}}{2} \tan \frac{\theta_{2}^{\prime}}{2}=\frac{\cos \frac{\beta+\alpha}{2}}{\cos \frac{\beta-\alpha}{2}}
$$

需要说明的是, 式(17)中相邻杆的外角 $\theta_{1}{ }^{\prime}$ 和 $\theta_{2}{ }^{\prime}$ 的作用区间为 $-180^{\circ} \leqslant \theta_{1}^{\prime} \leqslant 180^{\circ},-180^{\circ} \leqslant \theta_{2}^{\prime} \leqslant 180^{\circ}$, 而式(11) (16) 邻杆的外角 $\theta_{1}{ }^{\prime}$ 和 $\theta_{2}{ }^{\prime}$ 的作用区间为 $0^{\circ}$ $\leqslant \theta_{1}{ }^{\prime} \leqslant 360^{\circ}, 0^{\circ} \leqslant \theta_{2}^{\prime} \leqslant 360^{\circ}$ 。

用相邻杆内角 $\theta_{1} 、 \theta_{2}$ (图 6)表示正切积余弦表达 式为

$$
\tan \frac{\theta_{1}}{2} \tan \frac{\theta_{2}}{2}=\frac{\cos \frac{\beta-\alpha}{2}}{\cos \frac{\beta+\alpha}{2}}=k
$$

这种用相邻杆内角表示的正切积余弦表达式, 式中 $\alpha$ 和 $\beta$ 为锐角， $\theta_{1}$ 和 $\theta_{2}$ 的区间为 $-180^{\circ} \leqslant \theta_{1} \leqslant 180^{\circ}$, $-180^{\circ} \leqslant \theta_{2} \leqslant 180^{\circ}$ 。

在多环路 BNT 机构, 以及多环路 BNT 机构演 变而成的 Goldberg 机构中，使得各杆件之间的几何 关系变的非常直观、简明, 有重要的应用意义。

综上所述，由交错角 4 个区间得到的 4 组 BNT 机构, 本质上是相同的, 只是 1 组, 在使用中, 只 要选一组运动轴线方向都一致的 $\mathrm{R}_{41}\left(\mathrm{~S}_{41}\right)$ 区间就可 以了。

$\mathrm{R}_{41}$ 区间机构的 4 个运动副轴线的正方向都在 同一侧，并且轴线的正方向与相邻杆内角逆时针的 角位移方向一致。这个特点用于表示多环路 BNT 机构和多杆单环路 Goldberg 机构的角位移关系带来 了极大的方便。

由于 $\mathrm{S}_{41}$ 区第 4 象限和第 1 象限的交错角都可 以用锐角 $(-\pi / 2, \pi / 2)$ 方便地表示出来, 因此把 $S_{41}$ 区 间改写成用锐角表示的 $R_{41}$ 区间作为 BNT 机构交错 角的工作区间, 只要用锐角给出机构的结构参数方 程和闭环位移方程，就可以计算右旋型和左旋型两 种基本类型的 BNT 机构, 这样就取代了用钝角给出 的非线性安装和线性对称安装的两种不同形式的参 数方程。

因此， BNT 机构的两种基本类型(右旋型和 左旋型)的都是线性对称的，下面用交错角的锐角 给出的 BNT 机构的结构参数方程和闭环位移参 数方程。

4 交错角为锐角的 BNT 机构参数方程

\section{1 交错角为锐角的结构参数方程}

在给定 BNT 机构相邻杆交错角的锐角作用区 
间 $\mathrm{R}_{41}$ 为 $\left(-90^{\circ}<\alpha<90^{\circ}\right)$ 前提下, 机构封闭的几何条件 为: (1) 对边的杆长相等和交错角相等, 交错角的旋 向相同; (2) 相邻两边的边长比值与交错角的正弦值 比值相等, 符号相反; (3) 相邻两个边交错角的旋向 相反。

$$
\begin{gathered}
a_{1}=a_{3}=a \quad b_{2}=b_{4}=b \quad \alpha_{1}=\alpha_{3}=\alpha \quad \beta_{2}=\beta_{4}=\beta \\
\frac{a}{b}=-\frac{\sin \alpha}{\sin \beta}
\end{gathered}
$$

式(20)是相邻杆杆长比值和交错角正弦比值的关 系式。

$$
\boldsymbol{\tau}_{a}=-\boldsymbol{\tau}_{b} \quad\left|\boldsymbol{\tau}_{a}\right|=\left|\boldsymbol{\tau}_{b}\right|=1
$$

式中, $\boldsymbol{\tau}_{a}$ 和 $\boldsymbol{\tau}_{b}$ 是交错角的单位矢量。式(21)强调了 相邻两杆交错角旋向相反的特性, 也体现了它们不 能在作用区间的同一象限选取的约束条件。

\section{2 交错角为锐角的位移参数方程}

设 $\theta_{i j}$ 为第 $i$ 个杆件相对邻杆 $j$ 的角位移, 其中 $i=1,2,3,4, j=i-1$, 简写为 $\theta_{i}$ 。角位移 $\theta_{1} 、 \theta_{3}$ 和 $\theta_{2}$ 、 $\theta_{4}$ 都是相邻杆的内角。在 $\mathrm{R}_{41}$ 区间, $\theta_{i}(i=1,2,3,4)$ 的取值区间为 $-180^{\circ} \leqslant \theta_{i} \leqslant 180^{\circ}$ 。

BNT 机构用交错角为锐角表示的闭环位移参
数方程为

$$
\begin{gathered}
\theta_{1}=\theta_{3} \\
\theta_{2}=\theta_{4} \\
\tan \frac{\theta_{1}}{2} \tan \frac{\theta_{2}}{2}=\frac{\cos \frac{\beta-\alpha}{2}}{\cos \frac{\beta+\alpha}{2}}=k
\end{gathered}
$$

式(24)称之为相邻杆的内角余弦形正切积方程, $k$ 是 锐角余弦正切积常数。重要结论: 任何 BNT 机构都 是线性对称的机构。无论是机构的结构, 还是参数 方程都证明了这一结论是正确的。

相邻杆交错角的作用区间为 $\mathrm{R}_{41}\left(-90^{\circ}<\alpha<90^{\circ}\right)$ 时, 相邻杆交错角 $-90^{\circ}<\alpha<0^{\circ}, 0^{\circ}<\beta<90^{\circ}$, 得到右 旋型 BNT 机构; 相邻杆交错角 $0^{\circ}<\alpha<90^{\circ},-90^{\circ}<$ $\beta<0^{\circ}$, 得到左旋型 BNT 机构。式(20)和式(24)就 是 BNT 机构交错角为锐角的通用结构参数和位移 参数方程。

\begin{tabular}{|c|c|c|c|c|c|c|c|}
\hline \multicolumn{3}{|c|}{ 交错角的区间 } & $\alpha$ 的范围 & $\beta$ 的范围 & $\begin{array}{c}\text { 相邻杆长与交错角正弦 } \\
\text { 值的比值 }\end{array}$ & 外角正切积 & 内角正切积 \\
\hline \multirow{2}{*}{$\begin{array}{c}\mathrm{S}_{12} \\
(1-2 \\
\text { 象限 }) \\
\end{array}$} & \multicolumn{2}{|c|}{ 右旋型 } & $90^{\circ}<\alpha<180^{\circ}$ & $0^{\circ}<\beta<90^{\circ}$ & & & \\
\hline & \multicolumn{2}{|c|}{ 左旋型 } & $0^{\circ}<\alpha<90^{\circ}$ & $90^{\circ}<\beta<180^{\circ}$ & $\frac{a}{b}=\frac{\sin \alpha}{\sin \beta}$ & $\tan \frac{\theta_{1}^{\prime}}{2} \tan \frac{\theta_{2}^{\prime}}{2}=\frac{\sin \frac{\beta+\alpha}{2}}{\beta-\alpha}$ & $\tan \frac{\theta_{1}}{2} \tan \frac{\theta_{2}}{2}=\frac{\sin \frac{\beta-\alpha}{2}}{\beta+\alpha}$ \\
\hline \multirow{2}{*}{$\begin{array}{c}\mathrm{S}_{34} \\
(3-4 \\
\text { 象限) }\end{array}$} & \multicolumn{2}{|c|}{ 右旋型 } & $270^{\circ}<\alpha<360^{\circ}$ & $180^{\circ}<\beta<270^{\circ}$ & 适用于 $S_{12} 、 S_{34}$ 区间 & & $\sin \frac{1}{2}$ \\
\hline & \multicolumn{2}{|c|}{ 左旋型 } & $180^{\circ}<\alpha<270^{\circ}$ & $270^{\circ}<\beta<360^{\circ}$ & & 适用于 $S_{12} 、 S_{34}$ 区间 & 适用于 $S_{12} 、 S_{34}$ 区间 \\
\hline \multirow{3}{*}{$\begin{array}{l}\mathrm{S}_{23} \\
(2-3 \\
\text { 象限 }) \\
\end{array}$} & \multicolumn{2}{|c|}{ 右旋型 } & $90^{\circ}<\alpha<180^{\circ}$ & $180^{\circ}<\beta<270^{\circ}$ & \multirow{6}{*}{$\begin{array}{c}\frac{a}{b}=-\frac{\sin \alpha}{\sin \beta} \\
\text { 适用于 } \mathrm{S}_{23} 、 \mathrm{~S}_{41} \text { 区间, } \\
\text { 也适用于 } \mathrm{R}_{41} \text { 区间 }\end{array}$} & & \\
\hline & \multicolumn{2}{|c|}{ 左旋型 } & $180^{\circ}<\alpha<270^{\circ}$ & $90^{\circ}<\beta<180^{\circ}$ & & $\theta_{1}^{\prime} \quad \theta_{2}^{\prime} \quad \cos \frac{\beta+\alpha}{2}$ & $\theta_{2} \quad \cos \frac{\beta-\alpha}{2}$ \\
\hline & 右 & $\mathrm{S}_{41}$ & $270^{\circ}<\alpha<360^{\circ}$ & $0^{\circ}<\beta<90^{\circ}$ & & $\tan \frac{1}{2} \tan \frac{2}{2}=\frac{2}{\cos \frac{\beta-\alpha}{2}}$ & $\tan \frac{1}{2} \tan \frac{2}{2}=\frac{\beta}{\cos \frac{\beta+\alpha}{2}}$ \\
\hline \multirow{3}{*}{$\begin{array}{l}\mathrm{S}_{41} \text { 、 } \\
\mathrm{R}_{41} \\
(4-1 \\
\text { 象限) }\end{array}$} & $\begin{array}{l}\text { 旋 } \\
\text { 型 } \\
\end{array}$ & $\mathrm{R}_{41}$ & $-90^{\circ}<\alpha<0^{\circ}$ & $0^{\circ}<\beta<90^{\circ}$ & & 适用于 $\mathrm{S}_{23} 、 \mathrm{~S}_{41}$ 区间， & 适用于 $S_{23} 、 S_{41}$ 区间, \\
\hline & 左 & $\mathrm{S}_{41}$ & $0^{\circ}<\alpha<90^{\circ}$ & $270^{\circ}<\beta<360^{\circ}$ & & 也适用于 R 41 区间 & 也适用于 $\mathrm{R}_{41}$ 区间 \\
\hline & $\begin{array}{l}\text { 旋 } \\
\text { 型 }\end{array}$ & $\mathrm{R}_{41}$ & $0^{\circ}<\alpha<90^{\circ}$ & $-90^{\circ}<\beta<0^{\circ}$ & & & \\
\hline
\end{tabular}

表 1 给出了 BNT 机构相邻杆件交错角 4 个作用 区间和机构类型的关系。 $S_{12} 、 S_{23} 、 S_{34}$ 和 $S_{41}$ 区间都 可以转换成锐角的 $R_{41}$ 区间, 所以 $R_{41}$ 区间可以取代 它们，本质上只有一个 $\mathrm{R}_{41}$ 区间。

表 1 BNT 机构相邻杆件交错角作用区间与机构的类型的关系

\section{5 在多环路 BNT 机构中的应用}

图 14 是两个环路机架不共线的 5 杆两环右旋型 BNT 机构, 拆去两个环路公用的连架杆 6 , 就得到 图 15 机架不共线的右旋型 5 杆 Goldberg 机构。连 架杆 $1 、 4$ 和 6 的杆长 $a_{1}=a_{4}=a_{6}=120 \mathrm{~mm}$, 交错角 $\alpha_{1}=\alpha_{4}=\alpha_{6}=-10^{\circ}$ 。

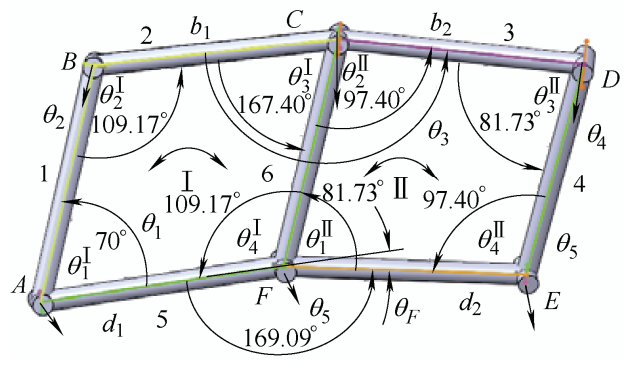

图 14 两环 BNT 机构 


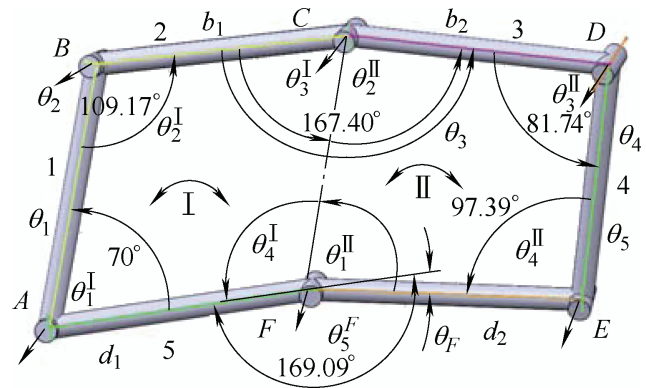

图 155 杆 Goldberg 机构

环路 I 连杆 $b_{1}$ 和机架 $d_{1}$ 的长度为 $b_{1}=d_{1}=120$ $\mathrm{mm}$, 交错角 $\beta_{1}=10^{\circ}$ 。环路 II 连杆 $b_{2}$ 和机架 $d_{2}$ 的长 度 $b_{2}=d_{2}=120 \mathrm{~mm}$, 交错角 $\beta_{2}=10^{\circ}$ 。因为 $\theta_{1}=\theta_{1}^{I}=\theta_{3}^{I}$, $\theta_{2}=\theta_{2}^{I}=\theta_{4}^{I}, \theta_{4}=\theta_{3}^{I I}=\theta_{1}^{I I}, \theta_{5}=\theta_{4}^{I I}=\theta_{2}^{I I}, \theta_{3}=\theta_{3}^{I}+\theta_{2}^{I I}$, 把 $\theta_{1}=\theta_{1}^{I}=70^{\circ}$ 代入式(24), 得到 $\tan \frac{70^{\circ}}{2} \tan \frac{\theta_{2}}{2}=\frac{\cos \frac{\beta_{1}-\alpha}{2}}{\cos \frac{\beta_{1}+\alpha}{2}}=\frac{\cos \frac{10-(-10)}{2}}{\cos \frac{10+(-10)}{2}}=\frac{\cos 10^{\circ}}{\cos 0^{\circ}}$

求得 $\theta_{2}=109.1736^{\circ} \approx 109.17^{\circ}=\theta_{4}^{I}$, 与图 14 和图 15 的实测结果一致。这个结果说明用交 错角为锐角建立的相邻构件内角的余弦正切 积公式(24)是正确的。

由图 15 可以非常直观地得出 5 杆 Goldberg 机构 的闭环位移方程，即

$$
\begin{gathered}
\theta_{3}-\theta_{1}-\theta_{5}=0 \\
\theta_{2}+\theta_{4}=2 \pi-\theta_{5}^{F} \\
\tan \frac{\theta_{1}}{2} \tan \frac{\theta_{2}}{2}=\frac{\cos \frac{\beta_{1}-\alpha}{2}}{\cos \frac{\beta_{1}+\alpha}{2}}=k_{1} \\
\tan \frac{\theta_{4}}{2} \tan \frac{\theta_{5}}{2}=\frac{\cos \frac{\beta_{2}-\alpha}{2}}{\cos \frac{\beta_{2}+\alpha}{2}}=k_{2}
\end{gathered}
$$

式(25 (28)为 4 个独立方程, 可以求解 5 个位移参 数 $\theta_{1} 、 \theta_{2} 、 \theta_{3} 、 \theta_{4}$ 和 $\theta_{5}$ 。把连架杆件 1 和件 5 作为输 入输出构件, 由式(27)和式(28)得到

$$
\tan \frac{\theta_{1}}{2} \tan \frac{\theta_{5}}{2}=k_{1} k_{2} /\left(-\tan \frac{\theta_{5}^{F}+\theta_{4}}{2} \tan \frac{\theta_{4}}{2}\right)
$$

这就是件 1 和件 5 的输入输出函数。因为 $\theta_{4}=\theta_{1}^{I I}=$ $360^{\circ}-\theta_{4}^{I}-\theta_{5}^{F}=360^{\circ}-109.17^{\circ}-109.09^{\circ}=81.74^{\circ}$, 计算 得到的 $\theta_{4}$ 与图 15 的 $\theta_{3}^{I I}\left(\theta_{4}\right)$ 的实测结果相同, 与图 14 的 $\theta_{3}^{I I}\left(\theta_{4} 、 \theta_{1}^{I I}\right)$ 的实测结果相同仅有 $0.01^{\circ}$ 的误差, 这里产生的 $0.01^{\circ}$ 误差是两次测量之间引起的误差, 显然可以忽略。把环路II的 $\theta_{4}=81.74^{\circ}$ 代入式(28), 得

$$
\tan \frac{81.74^{\circ}}{2} \tan \frac{\theta_{5}}{2}=\frac{\cos \frac{\beta_{2}-\alpha}{2}}{\cos \frac{\beta_{2}+\alpha}{2}}=\frac{\cos 10^{\circ}}{\cos 0^{\circ}}
$$

求得 $\theta_{5}=97.3910^{\circ} \approx 97.39^{\circ}$ 。说明用交错角为锐角建立 的相邻构件内角的余弦正切积公式(24)和式(28)是 正确的。

由图 14 和 15 都可以直观地得出 $\theta_{3}=\theta_{1}+\theta_{5}=70^{\circ}+$ $97.39^{\circ}=167.39^{\circ} \approx 167.40^{\circ}$ 。说明 5 杆 Goldberg 机构和 两环路 BNT 机构的运动学性能是等效的。把 $\theta_{1}=70^{\circ}$, $\theta_{5}^{F}=169.09^{\circ}, \theta_{4}=81.74^{\circ}$ 代入式(29), 则 $\tan \frac{70^{\circ}}{2} \tan \frac{\theta_{5}}{2}=\left(\frac{\cos 10^{\circ}}{\cos 0^{\circ}}\right)^{2} /\left(-\tan \frac{169.09^{\circ}+81.74^{\circ}}{2} \tan \frac{81.74^{\circ}}{2}\right)$ 求得 $\theta_{5}=97.395^{\circ} \approx 97.39^{\circ}$ 。 $\theta_{5}$ 的计算值和图 15 的检测 值相同, 说明式(29)是正确的。

以上的分析说明, 无论分析单环路BNT和多环 路BNT机构, 还是分析多环路BNT机构演变为单环 路的 Goldberg 机构、Myard 机构、6 杆 L 型 Goldberg 机构等, 采用内角的余弦正切积公式(24)都使得相 邻杆件之间的角位移几何关系变得简单、直观, 清 晰，非常便于应用。

\section{6 结论}

在对 BNT 机构杆件交错角的一周 $360^{\circ}$ 的分析 中, 由 $\mathrm{S}_{12}$ 和 $\mathrm{S}_{34}$ 区各得到 1 组非线性安装的左旋型 和右旋型两类机构; 由 $S_{23}$ 和 $S_{41}$ 区各得到 1 组线性 安装的左旋型和右旋型两类机构。通过对比发现, 2 组线性安装和 2 组非线性安装仅仅是运动副轴线不 对称, 机构的结构是完全对称的, 它们并没有本质 区别, 本质上只是 1 组机构。因此, 可以得出一个 重要的结论: BNT 机构只有左旋型和右旋型两类基 本机构, 它们都是线性对称的, 两种机构是一种运 动链, 它们可以用 $R_{41}$ 区间交错角为锐角的结构参 数和闭环位移参数方程统一表示。同时还得到下面 几个重要的规则。

(1) 交错角的象限选取约束条件为: 相邻杆的 交错角不能在作用区间的同一象限选取, 这一约束 条件是确保 BNT 机构环路封闭的必要约束之一。

(2) 相邻杆交错角的旋向相反。

（3） BNT 机构只有两种基本类型: 右旋型和左 旋型，这两种基本类型都是线性对称的。

（4）两类 BNT 机构, 都可以用交错角为锐角的 式(20)结构参数方程和式(24)闭环位移方程表示。

(5) 由图 5 和图 3、4, 以及图 6 11 的对比, 可以看出, 相邻杆的夹角用内角表示, 交错角的工 作区间采用锐角的 $\mathrm{R}_{41}\left(\mathrm{~S}_{41}\right)$ 区间，使得机构中各构 件的位移参数几何关系简单, 直观, 对分析多环路 组成的 BNT 机构将会带来很大的方便。 


\section{参 考 文 献}

[1] BENNETT G T. A new mechanism[J]. Engineering, 1903, 76: 777-778.

[2] BENNETT G T. The skew isogram mechanism[J]. Proceedings of the London Mathematical Society, 1914, 13: $151-173$.

[3] BAKER J E. The composition of Bennett's hyperboloids from the loop itself[J]. Journal of Mechanical Design, Transactions of the ASME, 2004, 126: 875-880.

[4] 黄真, 刘婧芳, 李艳文. 论机构自由度[M]. 北京: 科 学出版社, 2011.

HUANG Zhen, LIU Jingfang, LI Yanwen. Mobility analysis of mechanisms[M]. Beijing: Science Press, 2011.

[5] 王伟. 基于 Bennett 机构的空间大尺度可展网络的组装 与分析[D]. 哈尔滨：哈尔滨工业大学, 2011.

WANG Wei. Assembling and analysis of large special deployable networks based on Bennett linkage[D]. Harbin: Harbin Institute of Technology, 2011.

[6] HUANG Zhen, LIU Jingfang, ZENG Daxing. A general methodology for mobility analysis of mechanisms based on constraint screw theory[J]. Sci. China Ser. E-Tech. Sci., 2009, 52(5): 1337-1347.

[7] CHEN Yan, YOU Zhong. On mobile assemblies of Bennett linkages[J]. Proc. Royal Society A : Mathematical, Physical and Engineering Sciences, 2008, 464: 1275-1283.

[8] ZHANG Ketao, DAI J S. Screw-system-variation enabled reconfiguration of the Bennett plano-spherical hybrid linkage and its evolved parallel mechanism[J]. Journal of Mechanical Design, Trans. ASME, 2015, 137(6): 062303 .

[9] DENG Zongquan, HUANG Hailin, LI Bing, et al. Synthesis of deployable/foldable single loop mechanisms with revolute joints[J]. Journal of Mechanisms and Robotics, ASME, 2011, 3: 031006.

[10] YANG Fufu, CHEN Yan, KANG Rongjie, et al. Truss transformation method to obtain the non-overconstrained forms of 3D overconstrained linkages[J]. Mechanism and Machine Theory, 2016, 102: 149-166.

[11] CHEN Yan, PENG Rui, ZHONG You. Origami of thick panels[J]. Science Mag. Org., 2015， 349: 396-400.

[12］杨佳金金, 吕胜男, 丁希仑. 基于 Bennett 机构的柱面拟
合可展机构设计及分析 [J]. 深空探测学报, 2017, 4(4): 340-345.

YANG Jiaxin, LÜ Shengnan, DING Xilun. Design and analysis of approximate cylindrical deployable mechanism with Bennett linkages[J]. Journal of Deep Space Exploration, 2017, 4(4): 340-345.

[13] LIU Jingfang, YU Yueqing, HUANG Zen, et al. General order principle for multi-Bennett linkages[J]. Chinese Journal of Mechanical Engineering, 2013, 26(2): 275-281.

[14] BAKER J E. The Bennett, Goldberg and Myard linkages-in perspective[J]. Mechanism and Machine Theory, 1979, 14(4): 239-253.

[15] 张启先. 空间机构的分析与综合 [M]. 北京：机械工业 出版社, 1984.

ZHANG Qixian. Analysis and synthesis of spatial mechanism[M]. Beijing: China Machine Press, 1984.

[16] BAKER J E. Kinematic investigation of the deployable Bennett loop[J]. ASME J. Mech. Des, 2007, 129: 602-610.

[17] SONG C Y, CHEN Yan, CHEN I M. A 6R linkage reconfigurable between the line-symmetric Bricard linkage and the Bennett linkage[J]. Mechanism and Machine Theory, 2013, 70: 278-292.

[18] HERVÉ J M, DAHAN M. The two kinds of Bennett's mechanisms $[\mathrm{C}] / /$ Proceedings of the Sixth World Congress on Theory of Machines and Mechanisms, New Delhi, India, 1983: 116-119.

[19] CHEN Yan, BAKER J E. Using a Bennett linkage as a connector between other Bennett loops[J]. Proceeding of Institution of Mechanical Engineers , Journal of Multi-body Dynamics, 2005, 219(2): 177-185.

[20] BAKER J E. Introducing the supplementary Bennett loop $[\mathrm{J}]$. Mechanical Engineering Science, 2010, 225: 472-479.

作者简介: 鹿玲, 女, 1964 年出生, 副教授。主要研究方向为先进制造 工艺与机器人技术。

E-mail: luling@ysu.edu.cn 张一同(通信作者), 男, 1945 年出生, 教授。主要研究方向为机构结构 理论。

E-mail: ytzhang@ysu.edu.cn

牟德君, 女, 1967 年出生, 副教授。主要研究方向为机构结构理论。

E-mail: djmu@ysu.edu.cn

胡波, 男, 1982 年出生, 教授, 博士研究生导师。主要研究方向为机构 结构理论和机器人技术。

E-mail: hubo@ysu.edu.cn

卢文娟, 女, 1983 年出生, 副教授。主要研究方向为机构结构理论。

E-mail: luwenjuan@ysu.edu.cn 